\title{
On the derived dimension of abelian categories
}

\author{
Javad Asadollahi and Rasool Hafezi
}

\begin{abstract}
We give an upper bound on the dimension of the bounded derived category of an abelian category. We show that if $\mathcal{X}$ is a sufficiently nice subcategory of an abelian category, then the derived dimension of $\mathcal{A}$ is at most $\mathcal{X}-\operatorname{dim} \mathcal{A}$, provided that $\mathcal{X}-\operatorname{dim} \mathcal{A}$ is greater than one. We provide some applications.
\end{abstract}

\section{Introduction}

Motivated by the work of Bondal and Van den Berg [BV], Rouquier attached a numerical invariant to a triangulated category, its dimension. This dimension gives a new invariant for algebras and algebraic varieties under derived equivalences. Roughly speaking, it measures the minimum steps one requires to build the whole triangulated category $\mathcal{T}$ out of a single object $M$ (for details see [Rq2]). Rouquier's results enabled him to give the first example of Artin algebras of representation dimension greater than three (see [Rq1]). Therefore he could solve a long-standing open problem starting with Auslander's [Au] work in his Queen Mary notes from 1971.

The notion of dimension of triangulated categories then was considered by several authors and has found applications in different subjects ranging from algebraic geometry to representation theory of algebras and to commutative ring theory. In the setting of Artin algebras, it provides a lower bound for the representation dimension of an Artin algebra. Moreover, it is related to some known invariants like Lowey length and global dimension (see, e.g., [ABIM], [KK], [Opp], [O], [Rq1]).

Let $\mathcal{A}$ be an abelian category. It is shown by Han $[\mathrm{H}]$ that if $\mathcal{A}$ is of finite representation type, that is, if there exists an object $M$ in $\mathcal{A}$ with $\mathcal{A}=\operatorname{add} M$, then $\operatorname{dim} \mathbb{D}^{b}(\mathcal{A}) \leq 1$, where $\mathbb{D}^{b}(\mathcal{A})$ denotes the bounded derived category of $\mathcal{A}$. The invariant $\operatorname{dim} \mathbb{D}^{b}(\mathcal{A})$ is also known as the derived dimension of $\mathcal{A}$, denoted by $\operatorname{der} \operatorname{dim} \mathcal{A}$. Krause and Kussin [KK, Proposition 3.4] showed that if $\mathcal{A}$ is an abelian category with a representation generator $M$, then $\operatorname{dim} \mathbb{D}^{b}(\mathcal{A}) \leq$

Kyoto Journal of Mathematics, Vol. 54, No. 3 (2014), 693-702

DOI 10.1215/21562261-2693496, (C) 2014 by Kyoto University

Received January 16, 2013. Revised June 26, 2013. Accepted August 30, 2013.

2010 Mathematics Subject Classification: 18E10, 18E30, 18A40, 16E05, 16 G60.

Authors' research supported in part by grant no. 90130216 from Institute for Research in Fundamental Science (IPM). 
gl.dim $\operatorname{End}_{\mathcal{A}}(M)$. An add $M$-resolution of $X$ is an exact sequence $\cdots \rightarrow M_{n} \rightarrow$ $\cdots \rightarrow M_{1} \rightarrow M_{0} \rightarrow X \rightarrow 0$ such that $M_{i} \in$ add $M$, and the sequence is exact with respect to the functor $\operatorname{Hom}(M$,$) . An object M$ in $\mathcal{A}$ is called a representation generator if every object $X \in \mathcal{A}$ admits an add $M$-resolution of finite length.

In this note, we show that if $\mathcal{X}$ is a sufficiently nice subcategory of an abelian category $\mathcal{A}$, which is of finite representation type, then $\operatorname{dim} \mathbb{D}^{b}(\mathcal{A}) \leq \mathcal{X}$-dim $\mathcal{A}$, provided $\mathcal{X}-\operatorname{dim} \mathcal{A}>1$. As applications, we obtain a generalization of the main theorem of $[\mathrm{H}]$, provide a simple proof for [B, Corollary 6.7] (with some weaker conditions) and deduce [KK, Proposition 2.6] as a corollary. Furthermore, we get an upper bound on the derived dimension of $n$-torsionless finite algebras.

\section{Preliminaries}

SETUP

Throughout we assume that $\mathcal{A}$ is an abelian category. We denote the category of complexes over $\mathcal{A}$ by $\mathbb{C}(\mathcal{A})$. We grade the complexes cohomologically, so an object $A=\left(A^{n}, f^{n}\right)$ of $\mathbb{C}(\mathcal{A})$ will be written as

$$
A=\left(A^{n}, f^{n}\right): \cdots \longrightarrow A^{n-1} \stackrel{f^{n-1}}{\longrightarrow} A^{n} \stackrel{f^{n}}{\longrightarrow} A^{n+1} \longrightarrow \cdots
$$

For any integer $n$, we set $Z^{n} A:=\operatorname{Ker} f^{n}$ and $B^{n} A:=\operatorname{Im} f^{n-1}$.

\section{1 .}

Dimension of triangulated categories. Let $\mathcal{T}$ be a triangulated category, and let $\mathcal{I}_{1}$ and $\mathcal{I}_{2}$ be subcategories of $\mathcal{T}$. Using the same notation as in [Rq2, Section 3.1], we let $\mathcal{I}_{1} * \mathcal{I}_{2}$ denote the full subcategory of $\mathcal{T}$ consisting of objects $M$ such that there exists an exact triangle

$$
I_{1} \longrightarrow M \longrightarrow I_{2} \leadsto
$$

in $\mathcal{T}$ with $I_{i} \in \mathcal{I}_{i}$, for $i=1,2$.

Let $\mathcal{I}$ be a subcategory of $\mathcal{T}$. Denote by $\langle\mathcal{I}\rangle$ the smallest full subcategory of $\mathcal{T}$ that contains $\mathcal{I}$ and is closed under taking finite coproducts, direct factors, and all shifts. Finally, set $\mathcal{I}_{1} \diamond \mathcal{I}_{2}:=\left\langle\mathcal{I}_{1} * \mathcal{I}_{2}\right\rangle$. The octahedral axiom implies that the operations $*$ and $\diamond$ are associative (see $[\mathrm{BV}$, Section 2]).

Now, inductively one defines $\langle\mathcal{I}\rangle_{0}=0$ and $\langle\mathcal{I}\rangle_{n}=\langle\mathcal{I}\rangle_{n-1} \diamond\langle\mathcal{I}\rangle$ for $n \geq 1$.

\section{DEFINITION 2.2 ([Rq2, DEFINITION 3.2])}

Let $\mathcal{T}$ be a triangulated category. The dimension of $\mathcal{T}$, denoted by $\operatorname{dim} \mathcal{T}$, is defined by

$$
\operatorname{dim} \mathcal{T}=\inf \left\{d \in \mathbb{N} \mid \text { there exists } M \in \mathcal{T} \text { such that } \mathcal{T}=\langle M\rangle_{d+1}\right\} .
$$

We set $\operatorname{dim} \mathcal{T}=\infty$, if such $M$ does not exist.

\section{3.}

Let $\mathcal{M}$ be a class of objects of an abelian category $\mathcal{A}$. We denote by add $\mathcal{M}$ the objects that are isomorphic to a direct summand of a finite direct sum of objects 
in $\mathcal{M}$. If $\mathcal{M}=\{M\}$ contains a single object, we write add $M$ instead of add $\{M\}$. We say that a subcategory $\mathcal{X}$ of $\mathcal{A}$ is representation-finite if there exists an object $M \in \mathcal{X}$ such that add $\mathcal{X}=\operatorname{add} M$.

\section{Derived dimension of an abelian category}

Let us begin with a definition.

\section{DEFINITION 3.1}

Let $\mathcal{X}$ be a full subcategory of $\mathcal{A}$. For any object $A \in \mathcal{A}$, we define the $\mathcal{X}$ dimension of $A$, denoted $\mathcal{X}$ - $\operatorname{dim} A$, as follows. If $A \in \mathcal{X}$, then we set $\mathcal{X}$-dim $A=0$. If $t \geq 1$, then we set $\mathcal{X}$-dim $A \leq t$ if there exists an exact sequence

$$
0 \rightarrow X_{t} \rightarrow \cdots \rightarrow X_{0} \rightarrow A \rightarrow 0,
$$

where $X_{i} \in \mathcal{X}$ for $0 \leq i \leq t$. We set $\mathcal{X}$-dim $A=t$ if $\mathcal{X}$-dim $A \leq t$ and $\mathcal{X}$-dim $A \leq$ $t-1$. Finally if $\mathcal{X}$-dim $A \neq t$ for any $t \geq 0$, we set $\mathcal{X}$-dim $A=\infty$. The $\mathcal{X}$-global dimension of $A$, denoted $\mathcal{X}$-dim $\mathcal{A}$, is defined to be $\sup \{\mathcal{X}$-dim $A \mid A \in \mathcal{A}\}$.

Let $\mathcal{A}$ be an abelian category with enough projectives. A subcategory $\mathcal{X}$ of $\mathcal{A}$ is said to be resolving if it contains the projective objects and is closed under extensions and kernels of epimorphisms. For our purpose in this section, some weaker conditions are enough.

\section{DEFINITION 3.2}

Let $\mathcal{A}$ be an abelian category with enough projectives. A full subcategory $\mathcal{X} \subseteq \mathcal{A}$ is called semiresolving if it contains projective objects and satisfies the following property: for any short exact sequence $0 \rightarrow K \rightarrow P \rightarrow M \rightarrow 0$ in $\mathcal{A}$ with $P \in \operatorname{Prj} A$, if $M \in \mathcal{X}$, then so is $K$; otherwise, $\mathcal{X}$-dim $K=\mathcal{X}$-dim $M-1$.

It is clear that any resolving subcategory of $\mathcal{A}$ is semiresolving.

\section{NOTATION}

Let $\mathcal{A}$ be an abelian category. Given an object $M \in \mathcal{A}$ and an integer $i \in \mathbb{Z}$, we let $S^{i}(M)$ denote the complex with $M$ in the $i$ th place and 0 in the other places.

The following lemma can be proved using an argument similar to that in the proof of [KK, Lemma 2.5] (see also [C, Theorem 8.3]). So we skip the proof.

LEMMA 3.3

Let $\mathcal{X} \subseteq \mathcal{A}$ be a semiresolving subcategory of $\mathcal{A}$, where $\mathcal{A}$ is an abelian category with enough projectives. Let $d>0$ be an arbitrary integer. Let $A$ be a bounded complex in $\mathcal{A}$ such that for all $i$, both $\mathcal{X}-\operatorname{dim} B^{i} A$ and $\mathcal{X}-\operatorname{dim} Z^{i} A$ are at most $d$. Then there exists an exact triangle

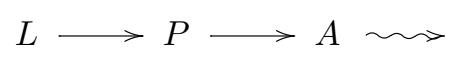


in $\mathbb{D}^{b}(\mathcal{A})$, in which $P$ is a complex of projectives and $L$ is a complex such that for all $i$, both $\mathcal{X}-\operatorname{dim} B^{i} L$ and $\mathcal{X}-\operatorname{dim} Z^{i} L$ are at most $d-1$. In fact, $P$ is a finite direct sum of complexes of the form $S^{i}(Q)$, where $i \in \mathbb{Z}$ and $Q$ is a projective object.

\section{THEOREM 3.4}

Let $\mathcal{X} \subseteq \mathcal{A}$ be a semiresolving subcategory of $\mathcal{A}$, where $\mathcal{A}$ is an abelian category with enough projectives. Assume that add $\mathcal{X}=\operatorname{add} M$, for some $M \in \mathcal{A}$. Let $A=$ $\left(A^{n}, f^{n}\right)$ be a bounded complex in $\mathcal{A}$ such that for all $i$, both $\mathcal{X}-\operatorname{dim} B^{i} A$ and $\mathcal{X}-\operatorname{dim} Z^{i} A$ are at most d. Then $A \in\langle\mathcal{M}\rangle_{d+2}$, where $\mathcal{M}=S^{0}(M)$.

\section{Proof}

We use induction on $d$. To begin, assume that $d=0$. As in [H, Theorem], consider the complexes $K=\left(Z^{n} A, 0\right)$ and $I=\left(B^{n+1} A, 0\right)$ to get the exact sequence

$$
0 \longrightarrow K \stackrel{i}{\longrightarrow} A \stackrel{\bar{f}}{\longrightarrow} I \rightarrow 0
$$

of complexes, where for each $n \in \mathbb{Z}, i_{n}: Z^{n} A \rightarrow A^{n}$ is the natural embedding and $\bar{f}^{n}: A^{n} \rightarrow B^{n+1} A$ is the map $f^{n}$ whose coimage is restricted to $B^{n+1} A$. So we get the triangle

$$
K \longrightarrow A \longrightarrow I \leadsto
$$

in $\mathbb{D}^{b}(\mathcal{A})$. Since $A$ is a bounded complex, $K=\bigoplus S^{n}\left(Z^{n} A\right)$ and $I=\bigoplus S^{n}\left(B^{n+1} A\right)$ have only finitely many nonzero summands. Hence by definition $K$ and $I$ belong to $\langle\mathcal{M}\rangle$. Thus $A \in\langle\mathcal{M}\rangle_{2}$. Assume inductively that $d>0$ and the result has been proved for values smaller than $d$. Lemma 3.3 implies the existence of an exact triangle

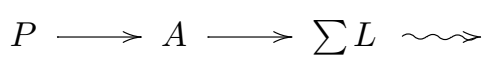

in $\mathbb{D}^{b}(\mathcal{A})$, where $P$ is a finite direct sum of stalk complexes of the form $S^{i}(Q)$, for some $i \in \mathbb{Z}$ and some projective objects $Q$, and $L$ is a complex such that $\mathcal{X}$-dim $B^{n} L \leq d-1$ and $\mathcal{X}$-dim $Z^{n} L \leq d-1$ for all $n$. Since $\mathcal{X}$ is semiresolving, $P \in\langle\mathcal{M}\rangle$. Moreover, by the induction hypothesis, $\sum L \in\langle\mathcal{M}\rangle_{d+1}$. Hence

$$
A \in\left\langle\langle\mathcal{M}\rangle *\langle\mathcal{M}\rangle_{d+1}\right\rangle \text {. }
$$

Now the associativity of the operation $*$ implies that

$$
\left\langle\langle\mathcal{M}\rangle *\langle\mathcal{M}\rangle_{d+1}\right\rangle=\left\langle\langle\mathcal{M}\rangle_{d+1} *\langle\mathcal{M}\rangle\right\rangle .
$$

So $A \in\langle\mathcal{M}\rangle_{d+2}$. This completes the inductive step and hence the proof.

Here we present two applications of the above theorem.

(1) Let $\mathcal{A}$ be an abelian category. It is proved by Han $[\mathrm{H}]$ that if there exists $M \in \mathcal{A}$ such that $\mathcal{A}=\operatorname{add} M$, then $\operatorname{dim} \mathbb{D}^{b}(\mathcal{A}) \leq 1$. In the following we Theorem 3.4 to provide a generalization of this result. 


\section{PROPOSITION 3.5}

Let $\mathcal{X} \subseteq \mathcal{A}$ be a representation-finite semiresolving subcategory of $\mathcal{A}$. Then $\operatorname{dim} \mathbb{D}^{b}(\mathcal{A}) \leq \mathcal{X}-\operatorname{dim} \mathcal{A}+1$

Proof

If $\mathcal{X}-\operatorname{dim} \mathcal{A}=\infty$, there is nothing to prove. So assume that $\mathcal{X}$ - $\operatorname{dim} \mathcal{A}$ is finite, say, $d$. So for any complex $A=\left(A_{n}, f_{n}\right)$ in $\mathcal{A}$, we have $\mathcal{X}$-dim $Z^{i} X \leq d$ and $\mathcal{X}$-dim $B^{i} X \leq d$. Assume that add $\mathcal{X}=$ add $M$, for some $M \in \mathcal{A}$. The above theorem now applies to show that $A \in\langle M\rangle_{d+2}$. Therefore $\mathbb{D}^{b}(\mathcal{A})=\langle M\rangle_{d+2}$, since $A$ was an arbitrary bounded complex.

(2) Let $\Omega^{d}(\mathcal{A})$ be the full subcategory of $\mathcal{A}$ consisting of all $d$ th syzygies, that is, all objects $A$ for which there exists an exact sequence

$$
0 \rightarrow A \rightarrow P^{0} \rightarrow \cdots \rightarrow P^{d-1}
$$

where $P^{i}$, for $0 \leq i \leq d-1$, is projective. Note that by definition, $\Omega^{d}(\mathcal{A})$ contains projectives and $\Omega^{d}(\mathcal{A})$ - $\operatorname{dim} \mathcal{A}=d$. Although we do not know whether $\Omega^{d}(\mathcal{A})$ is semiresolving or not, the proof of the above theorem can still be rewritten for this special case, and so we may prove the following corollary. We leave the details to the reader.

\section{COROLLARY 3.6}

Let $\mathcal{A}$ be an abelian category with enough projectives. Assume that $\Omega^{d}(\mathcal{A})$ is representation-finite. Then $\operatorname{dim} \mathbb{D}^{b}(\mathcal{A}) \leq d+1$.

\section{REMARK 3.7}

We just remark that in this case when $\Lambda$ is an Artin algebra and $\mathcal{A}=\bmod \Lambda$, then $\Omega^{d}(\mathcal{A})$ is known as the subcategory of $\bmod \Lambda$ consisting of $d$-torsionless modules. We say that $\Lambda$ is a $d$-torsionless-finite algebra provided that $\Omega^{d}(\mathcal{A})$ is representation-finite. The above corollary, in particular, shows that the derived dimension of $d$-torsionless-finite algebras is at most $d+1$.

Our aim now is to show that, if $\mathcal{X}-\operatorname{dim} \mathcal{A} \geq 2$, we may get a sharper upper bound for the dimension of the derived category of $\mathcal{A}$. To this end we recall the notion of a relative derived category.

As above, $\mathcal{A}$ is an abelian category. A complex $A$ in $\mathcal{A}$ is called $\mathcal{X}$-acyclic (sometimes $\mathcal{X}$-proper exact) if, for any $C \in \mathcal{X}$, the induced complex $\mathcal{A}(C, A)$ of abelian groups is acyclic. A chain map $f: A \longrightarrow B$ of complexes is called an $\mathcal{X}$ quasi-isomorphism if the induced chain map $\mathcal{A}(C, f)$ is a quasi-isomorphism for each $C \in \mathcal{X}$.

Let $\mathbb{K}(\mathcal{A})$ denote the homotopy category of $\mathcal{A}$ and let $\mathbb{K}^{-}(\mathcal{A})$ (resp., $\mathbb{K}^{b}(\mathcal{A})$ ) denote the full triangulated subcategory of $\mathbb{K}(\mathcal{A})$ consisting of all bounded above (resp., bounded) complexes. 
Assume that $\mathcal{X} \subseteq \mathcal{A}$ is a contravariantly finite subcategory. Set $\mathbb{K}_{\mathcal{X} \text {-ac }}^{*}(\mathcal{A}):=$ $\left\{X \in \mathbb{K}^{*}(\mathcal{A}) \mid X\right.$ is $\mathcal{X}$-acyclic $\}$, where $* \in\{$ blank,,$- b\}$. Since $\mathbb{K}_{\mathcal{X} \text {-ac }}^{*}(\mathcal{A})=\mathcal{X}^{\perp}$, it is a thick triangulated subcategory of $\mathbb{K}^{*}(\mathcal{A})$. Moreover, $f: X \rightarrow Y$ is an $\mathcal{X}$ quasi-isomorphism if and only if its mapping cone cone $(f)$ is $\mathcal{X}$-acyclic. Using this fact, similarly to [GZ, Lamma 2.3], one may deduce that the corresponding compatible saturated multiplicative system of $\mathbb{K}_{\mathcal{X}-\text { ac }}^{*}(\mathcal{A})$ is the collection of all $\mathcal{X}$-quasi-isomorphisms in $\mathbb{K}^{*}(\mathcal{A})$. Therefore, one gets a triangulated category by forming the quotient category $\mathbb{K}^{*}(\mathcal{A}) / \mathbb{K}_{\mathcal{X} \text {-ac }}^{*}(\mathcal{A})$, where $* \in\{$ blank,,$- b\}$. This category is called the $\mathcal{X}$-relative derived category of $\mathcal{A}$ and is denoted by $\mathbb{D}_{\mathcal{X}}^{*}(\mathcal{A})$ (see [Ch, Section 3]).

Note that if $\mathcal{A}$ has enough projective objects and $\mathcal{X}=\operatorname{Prj} \mathcal{A}$, then $\mathbb{D}_{\mathcal{X}}^{*}(\mathcal{A})$ is the usual derived category of $\mathcal{A}, \mathbb{D}^{*}(\mathcal{A})$. Furthermore, if $\mathcal{A}$ has enough projective objects and $\mathcal{X}=\operatorname{GPrj}(\mathcal{A})$ is the class of all Gorenstein projective objects of $\mathcal{A}$, then $\mathbb{D}_{\mathcal{X}}^{*}(\mathcal{A})$ is known as the Gorenstein derived category of $\mathcal{A}$, introduced and studied in [GZ], denoted by $\mathbb{D}_{\mathcal{G} \mathcal{P}}^{*}(\mathcal{A})$. Note that $\mathbb{K}_{\mathcal{X}-\text { ac }}^{*}(\mathcal{A})=\mathbb{K}_{\text {gpac }}^{*}(\mathcal{A})$ is the class of all Gorenstein projective proper exact sequences.

Let $\mathbb{K}^{-, \mathcal{X} b}(\mathcal{X})$ denote the full subcategory of $\mathbb{K}^{-}(\mathcal{X})$ consisting of all complexes $\mathbf{X} \in \mathbb{K}^{-}(\mathcal{X})$ such that for any $X \in \mathcal{X}$, the induced complex $\mathcal{A}(X, \mathbf{X})$ is acyclic, in degrees small enough, that is, $\mathrm{H}^{i} \mathcal{A}(X, \mathbf{X})=0$, for all $i \ll 0$.

\section{PROPOSITION 3.8}

Let $\mathcal{X} \subseteq \mathcal{A}$ be a contravariantly finite subcategory of $\mathcal{A}$. Then

$$
\mathbb{D}_{\mathcal{X}}^{b}(\mathcal{A}) \simeq \mathbb{K}^{-, \mathcal{X} b}(\mathcal{X})
$$

\section{Proof}

The argument is similar to the proof of [GZ, Theorem 3.6(ii)]. So we just provide an outline and refer the reader to that paper for details. Denote the composition of the functors $\mathbb{K}^{-, \mathcal{X} b}(\mathcal{X}) \hookrightarrow \mathbb{K}^{-}(\mathcal{A})$ and $\mathbb{K}^{-}(\mathcal{A}) \rightarrow \mathbb{D}_{\mathcal{X}}^{-}(\mathcal{A})$ by $F: \mathbb{K}^{-, \mathcal{X} b}(\mathcal{X}) \rightarrow$ $\mathbb{D}_{\mathcal{X}}^{-}(\mathcal{A})$. The proof of $[\mathrm{GZ}$, Theorem 3.6(ii)] applies to show that the image of $F$ is in $\mathbb{D}_{\mathcal{X}}^{b}(\mathcal{A})$, and so $F$ induces a functor $\mathbb{K}^{-, \mathcal{X}^{b}}(\mathcal{X}) \rightarrow \mathbb{D}_{\mathcal{X}}^{b}(\mathcal{A})$, which we denote again by $F$. Since $\mathcal{X}$ is contravariantly finite, an argument similar to the proof of [GZ, Proposition 3.4(ii)] implies that $F$ is dense. The fact that $F$ is fully faithful follows using arguments similar to [GZ, Propositions 2.7, 2.8].

Note that since a complex is $\mathcal{X}$-acyclic if and only if it is (add $\mathcal{X})$-acyclic, $\mathbb{K}^{-, \mathcal{X} b}(\mathcal{X})$ is a triangulated subcategory of $\mathbb{K}^{-,(\operatorname{add} \mathcal{X}) b}(\operatorname{add} \mathcal{X})$. An argument similar to [Ric, Lemma 2.2] applies to show that in fact we have an equivalence $\mathbb{K}^{-, \mathcal{X} b}(\mathcal{X}) \simeq$ $\mathbb{K}^{-,(\operatorname{add} \mathcal{X}) b}(\operatorname{add} \mathcal{X})$, as triangulated categories. We use this equivalence in the proof of the following theorem.

\section{THEOREM 3.9}

Let $\mathcal{X}$ be a contravariantly finite subcategory of $\mathcal{A}$ such that $\operatorname{add} \mathcal{X}=\operatorname{add} M$, for some $M \in \mathcal{A}$. Set $\Gamma=\operatorname{End}(M)$. Then $\mathbb{D}_{\mathcal{X}}^{b}(\mathcal{A}) \simeq \mathbb{D}^{b}(\Gamma)$. 
Proof

By the above proposition, $\mathbb{D}_{\mathcal{X}}^{b}(\mathcal{A}) \simeq \mathbb{K}^{-, \mathcal{X} b}(\mathcal{X})$. Moreover,

$$
\mathbb{K}^{-, \mathcal{X} b}(\mathcal{X}) \simeq \mathbb{K}^{-,(\operatorname{add} \mathcal{X}) b}(\operatorname{add} \mathcal{X}) .
$$

By assumption $\mathbb{K}^{-,(\operatorname{add} \mathcal{X}) b}(\operatorname{add} \mathcal{X}) \simeq \mathbb{K}^{-,(\operatorname{add} M) b}(\operatorname{add} M)$. On the other hand, it is known that the functor $\operatorname{Hom}_{\mathcal{A}}(M,-): \operatorname{add} M \rightarrow \operatorname{prj} \Gamma$ is an equivalence. This functor can be extended naturally to an equivalence of triangulated categories between $\mathbb{K}^{-,(\operatorname{add} M) b}(\operatorname{add} M)$ and $\mathbb{K}^{-, b}(\operatorname{prj} \Gamma)$ (see $[$ Ha, p. 108] for an absolute version of this fact). Finally, it is well known that $\mathbb{K}^{-, b}(\operatorname{prj} \Gamma) \simeq \mathbb{D}^{b}(\Gamma)$. This completes the proof.

PROPOSITION 3.10

Let $\mathcal{X}$ be a semiresolving contravariantly finite subcategory of $\mathcal{A}$ such that add $\mathcal{X}=$ add $M$, for some $M \in \mathcal{A}$. Set $\Gamma=\operatorname{End}(M)$. Then

$$
\operatorname{dim} \mathbb{D}_{\mathcal{X}}^{b}(\mathcal{A}) \leq \max \{2, \mathcal{X}-\operatorname{dim} \mathcal{A}\}
$$

\section{Proof}

In view of Theorem 3.9 we have $\operatorname{dim} \mathbb{D}_{\mathcal{X}}^{b}(\mathcal{A})=\operatorname{dim} \mathbb{D}^{b}(\Gamma)$. By [KK, Proposition 2.6], $\operatorname{dim} \mathbb{D}^{b}(\Gamma) \leq$ gl.dim $\Gamma$. So to complete the proof, it is enough to show that gl.dim $\Gamma \leq \max \{2, \mathcal{X}-\operatorname{dim} \mathcal{A}\}$. But this follows using techniques similar to those in [B, Proposition 6.4].

\section{THEOREM 3.11}

Let $\mathcal{X} \subseteq \mathcal{A}$ be a representation-finite semiresolving subcategory of $\mathcal{A}$. Set $\mathcal{X}$ $\operatorname{dim} \mathcal{A}=d$. Then

(i) If $d=0$ or 1 , then $\operatorname{dim} \mathbb{D}^{b}(\mathcal{A}) \leq d+1$.

(ii) If $\mathcal{X}$ is contravariantly finite and $d \geq 2$, then $\operatorname{dim} \mathbb{D}^{b}(\mathcal{A}) \leq d$.

\section{Proof}

(i) If $\mathcal{X}$ - $\operatorname{dim} \mathcal{A}$ is either zero or one, the result follows from Proposition 3.5.

(ii) Let $\mathcal{X}$ - $\operatorname{dim} \mathcal{A}=d \geq 2$. Assume that add $\mathcal{X}=\operatorname{add} M$. Since $\mathcal{X}$ contains projectives, any $\mathcal{X}$-acyclic complex is acyclic, and so $\mathbb{K}_{\mathcal{X} \text { - ac }}^{b}(\mathcal{A}) \subseteq \mathbb{K}_{\mathrm{ac}}^{b}(\mathcal{A})$. This, in turn, implies that there exists a quotient functor $\mathbb{D}_{\mathcal{X}}^{b}(\mathcal{A}) \longrightarrow \mathbb{D}^{b}(\mathcal{A})$. Therefore $\operatorname{dim} \mathbb{D}^{b}(\mathcal{A}) \leq \operatorname{dim} \mathbb{D}_{\mathcal{X}}^{b}(\mathcal{A})$. Now the result follows from the above proposition.

Note that in the case where $\mathcal{A}(A, B)$ is finitely generated, for any two objects $A$ and $B$ of $\mathcal{A}$, any representation-finite subcategory is automatically contravariantly finite. So the assumption in statement (ii) of Theorem 3.11 will be redundant.

Toward the end of the paper, we provide two applications of the above theorem (cf. [B, Corollary 6.7]). 
(1) Let $\mathcal{A}$ be an abelian category with enough projective objects. An object $G \in \mathcal{A}$ is called Gorenstein-projective if there exists an exact sequence

$$
\cdots \rightarrow P_{1} \rightarrow P_{0} \rightarrow P_{-1} \rightarrow \cdots
$$

of projective objects such that the sequence remains exact under the functor $\mathcal{A}(, Q)$, for any projective object $Q$ and $G \cong \operatorname{Ker}\left(P_{0} \rightarrow P_{-1}\right)$. In module categories, these modules were introduced by Auslander and Bridger in [AB], over commutative Noetherian rings, where they called them modules of Gorenstein dimension zero. They have been generalized to arbitrary modules over arbitrary rings by Enochs and Jenda [EJ]. They form the building blocks of a branch of homological algebra, known as Gorenstein homological algebra.

Let $\operatorname{GPrj}(\mathcal{A})$ denotes the class of all Gorenstein-projective objects. It is clear that this class contains projective objects. In fact, it is easy to see that $\operatorname{GPrj}(\mathcal{A})$ is a resolving subcategory of $\mathcal{A}$. For a proof of this fact in the module case see [AM]. One can use this class to attach a homological dimension to any object $A$, namely, the Gorenstein-projective dimension of $A$, denoted by $\operatorname{Gpd} A$. The Gorenstein global dimension of $\mathcal{A}$ is defined to be the supremum of the Gorenstein-projective dimension of all objects and will be denoted by $\operatorname{Ggldim} \mathcal{A}$. The above theorem implies that

$$
\operatorname{dim} \mathbb{D}^{b}(\mathcal{A}) \leq \max \{2, \operatorname{Ggl} \operatorname{dim} \mathcal{A}\},
$$

provided that $\operatorname{GPrj}(\mathcal{A})=\operatorname{add} A$, for some $A \in \mathcal{A}$.

(2) An important special case of (i) is $\mathcal{A}=\bmod \Lambda$, where $\Lambda$ is an Artin algebra and $\bmod \Lambda$ denotes the full subcategory of $\operatorname{Mod} \Lambda$ consisting of all finitely presented $\Lambda$-modules. Set $\operatorname{Gprj} \Lambda=\operatorname{GPrj} \Lambda \cap \bmod \Lambda$.

\section{DEFINITION 3.12}

An Artin algebra $\Lambda$ is said to be of finite Cohen-Macaulay type, finite CM-type for short, if the full subcategory $\operatorname{Gprj} \Lambda$ is of finite representation type.

If $\Lambda$ is a self-injective algebra, then it is of finite CM-type if and only if it is of finite representation type. Recall that an Artin algebra $\Lambda$ is called Gorenstein if $\operatorname{id}_{\Lambda} \Lambda<\infty$ and $\operatorname{id} \Lambda_{\Lambda}<\infty$. In this case it is known that $\operatorname{id}_{\Lambda} \Lambda=\operatorname{id} \Lambda_{\Lambda}$. If we let this common value be $d$, then we call $\Lambda$ a $d$-Gorenstein algebra. It is known that if $\Lambda$ is a $d$-Gorenstein algebra, then $\Omega^{d}(\bmod \Lambda)=\operatorname{Gprj} \Lambda$. So we get the following result.

COROLLARY 3.13 ([B, COROLLARY 6.7])

Let $\Lambda$ be a d-Gorenstein Artin algebra of finite CM-type. Then $\operatorname{dim} \mathbb{D}^{b}(\Lambda) \leq$ $\max \{2, d\}$.

Proof

To prove the corollary, one just should note that by our assumptions $\Omega^{d}(\bmod \Lambda)=$ Gprj $\Lambda$. Now the result follows from Theorem 3.11. 
Acknowledgments. We would like to thank the referees for their useful comments and pointing out a mistake in the first version of the paper. Ryo Takahashi mentioned that he, T. Aihara, T. Araya, O. Iyama, and M. Yoshiwaki also have a proof for Theorem 3.11 (see [AATY]). Their approach is completely different from ours. We thank them for letting us have the preliminary version of their preprint. The authors thank the Center of Excellence for Mathematics (University of Isfahan, Iran).

\section{References}

[AATY] T. Aihara, T. Araya, O. Iyama, R. Takahashi, and M. Yoshiwaki, Dimensions of triangulated categories with respect to subcategories, J. Algebra 339 (2014), 205-219.

[Au] M. Auslander, The Representation Dimension of Artin Algebras, Queen Mary College Mathematics Notes (1971), republished in Selected works of Maurice Auslander, Amer. Math. Soc., Providence, 1999.

[AB] M. Auslander and M. Bridger, Stable Module Theory, Mem. Amer. Math. Soc. 94, Amer. Math. Soc., Providence, 1969. MR 0269685.

[ABIM] L. L. Avramov, R. Buchweitz, S. Iyengar, and C. Miller, Homology of perfect complexes, Adv. Math. 223 (2010), 1731-1781. MR 2592508. DOI 10.1016/j.aim.2009.10.009.

[AM] L. L. Avramov and A. Martsinkovsky, Absolute, relative, and Tate cohomology of modules of finite Gorenstein dimension, Proc. London Math. Soc. (3) 85 (2002), 393-440. MR 1912056.

DOI 10.1112/S0024611502013527.

[B] A. Beligiannis, On algebras of finite Cohen-Macaulay type, Adv. Math. 226 (2011), 1973-2019. MR 2737805. DOI 10.1016/j.aim.2010.09.006.

[BV] A. Bondal and M. van den Bergh, Generators and representability of functors in commutative and noncommutative geometry, Mosc. Math. J. 3 (2003), no. 1, 1-36. MR 1996800.

[Ch] X.-W. Chen, Homotopy equivalences induced by balanced pairs, J. Algebra 324 (2010), 2718-2731. MR 2725197. DOI 10.1016/j.jalgebra.2010.09.002.

[C] J. D. Christensen, Ideals in triangulated categories: Phantoms, ghosts, and skeleta, Adv. Math. 136 (1998), 284-339. MR 1626856.

DOI 10.1006/aima.1998.1735.

[EJ] E. Enochs and O. M. G. Jenda, Gorenstein injective and projective modules, Math. Z. 220 (1995), 611-633. MR 1363858. DOI 10.1007/BF02572634.

[GZ] N. Gao and P. Zhang, Gorenstein derived categories, J. Algebra 323 (2010), 2041-2057. MR 2594663. DOI 10.1016/j.jalgebra.2010.01.027.

[H] Y. Han, Derived dimensions of representation-finite algebras, preprint, arXiv:0909.0330v1 [math.RT].

[Ha] D. Happel, Triangulated Categories in the Representation Theory of Finite-Dimensional Algebras, London Math. Soc. Lecture Note Ser. 119, 
Cambridge Univ. Press, Cambridge, 1988. MR 0935124.

DOI 10.1017/CBO9780511629228.

[KK] H. Krause and D. Kussin, "Rouquier's theorem on representation dimension" in Trends in Representation Theory of Algebras and Related Topics, Contemp. Math. 406, Amer. Math. Soc., Providence, 2006, 95-103. MR 2258043. DOI 10.1090/conm/406/07655.

[Opp] S. Oppermann, Lower bounds for Auslander's representation dimension, Duke Math. J. 148 (2009), 211-249. MR 2524495.

DOI 10.1215/00127094-2009-025.

[O] D. Orlov, Remarks on generators and dimensions of triangulated categories, Moscow Math. J. 9 (2009) no. 1, 153-159. MR 2567400.

[Ric] J. Rickard, Morita theory for derived category, J. London Math. Soc. (2) 39 (1989), 436-456. MR 1002456. DOI 10.1112/jlms/s2-39.3.436.

[Rq1] R. Rouquier, Representation dimension of exterior algebras, Invent. Math. 165 (2006), 357-367. MR 2231960. DOI 10.1007/s00222-006-0499-7.

[Rq2] Dimension of triangulated categories, J. K-Theory 1 (2008), 193-256. MR 2434186. DOI 10.1017/is007011012jkt010.

Asadollahi: Department of Mathematics, University of Isfahan, Isfahan, Iran and School of Mathematics, Institute for Research in Fundamental Science (IPM), Tehran, Iran; asadollahi@ipm.ir

Hafezi: School of Mathematics, Institute for Research in Fundamental Science (IPM), Tehran, Iran; hafezi@ipm.ir 